\title{
Cytokine-like 1 is involved in the growth and metastasis of neuroblastoma cells
}

\author{
MINGJIE WEN ${ }^{1}$, HUANMIN WANG $^{2}$, XULONG ZHANG $^{1}$, JUN LONG $^{1}$, \\ ZHE LV ${ }^{1}$, QINGLI KONG ${ }^{1}$ and YUNQING AN ${ }^{1}$ \\ ${ }^{1}$ Department of Immunology, School of Basic Medical Sciences, Capital Medical University, Beijing 100069; \\ ${ }^{2}$ Department of Surgery, Beijing Children's Hospital, Capital Medical University, Beijing 100045, P.R. China
}

Received April 12, 2012; Accepted June 15, 2012

DOI: $10.3892 /$ ijo.2012.1552

\begin{abstract}
Cytokine-like 1 (CYTL1) was first identified in CD $34^{+}$cells derived from bone marrow and cord blood. The biological functions of CYTL1 remain largely unknown. Here, we reveal a relationship between CYTL1 expression and the biological characteristics of neuroblastoma (NB). The expression of CYTL1 was detected in 10 human tumor cell lines and human NB tissues by RT-PCR and real-time PCR. The inhibitory effect of CYTL1 knockdown on the proliferation, migration and invasion of SH-SY5Y human neuroblastoma cells was studied using the CCK-8 assay and Transwell chamber assays. Among the 10 human tumor cell lines that we examined, CYTL1 was expressed only in SH-SY5Y human neuroblastoma cells. Furthermore, we also observed high levels of CYTL1 expression in human NB tissues. When CYTL1 expression was blocked by siRNA, SH-SY5Y cells showed decreased proliferation, migration and invasion activities. Taken together, our results showed the first evidence of CYTL1 expression in SH-SY5Y neuroblastoma cells and human NB tissues, revealed a possible link between CYTL1 and NB development, and suggested CYTL1 as a potential therapeutic target and diagnosis biomarker for NB.
\end{abstract}

\section{Introduction}

Neuroblastoma (NB) is the third most common malignant solid tumor in early childhood, following leukemia and central nervous system tumors (1). This neuroendocrine tumor arises from the neural crest cells, which are the precursors of the sympathetic nervous system. Most NB deaths occur within 2 years of diagnosis. NB accounts for almost $15 \%$ of childhood cancer fatalities. Metastasis is the main cause of mortality in NB patients (2). NB tends to metastasize to the bone, bone marrow,

Correspondence to: Professor Yunqing An, Department of Immunology, School of Basic Medical Sciences, Capital Medical University, No. 10 Xitoutiao, You An Men, Beijing 100069, P.R. China E-mail: anyunq@ccmu.edu.cn

Key words: cytokine-like 1, neuroblastoma, siRNA, growth, metastasis liver, lymph nodes, and skin. At least $70 \%$ of affected patients exhibit disseminated disease at the time of diagnosis (3). Cytokines, chemokines, and their receptors, including VEGF, IL-6, CCL2/CCR2, CXCL12/CXCR4 and CX3CL/CX3CR1, have been found to be involved in the growth and metastasis of NB (4-8).

Cytokine-like 1 (CYTL1), also called C17, was first identified approximately 10 years ago in the bone marrow and cord blood mononuclear cells that bear the CD34 surface marker (9). Until recently, however, knowledge of CYTL1 has remained limited and has mainly focused on its function in cartilage development and arthritis (10-13). Recent studies have demonstrated that CYTL1 maintains cartilage homeostasis by functioning as an autocrine/paracrine factor and that deletion of the Cytll gene increases osteoarthritic cartilage destruction in Cytll knockout (Cytl1-/-) mice $(9,11,12)$. Another recent study has shown that CYTL1 prevents early stage inflammatory arthritis and its associated joint destruction but not disease progression. That study proposed that CYTL1 contributes to immune homeostasis systemically or in a tissue-specific manner in the joint (13). CYTL1 is also upregulated in benign prostatic hypertrophy (14) and is regulated by DNA methylation in human lung squamous cell carcinoma (SCC) (15). Recent reports have suggested that CYTL1 is more likely to adopt a CCL2-like chemokine folding structure to signal through the CCR2 receptor (16). Currently, there are no reports concerning the relationship between CYTL1 and NB.

In the present study, we examined CYTL1 expression in various human tumor cell lines, determined the expression levels of CYTL1 in NB tissues, and investigated a possible role of CYTL1 expression in the growth and metastasis of NB.

\section{Materials and methods}

Cell culture of 10 human tumor cell lines. The SH-SY5Y,MCF-7, HeLa and Raji cell lines used in this study were maintained in Dulbecco's modified Eagle's medium (DMEM) (Hyclone, Logan, UT, USA) supplemented with $10 \%$ fetal bovine serum (FBS) (Hyclone). The A549, MDA-MB-231, 7402, HepG2, SW480 and SiHa cell lines were cultured in RPMI-1640 medium (Gibco-BRL, Carlsbad, CA, USA) supplemented with $10 \%$ FBS (Hyclone). All cells were cultured at $37^{\circ} \mathrm{C}$ in a humidified atmosphere containing $5 \% \mathrm{CO}_{2}$. After culturing, cells 
Table I. The primer sequences of target genes.

\begin{tabular}{llllc}
\hline Gene & GenBank ID & \multicolumn{1}{c}{ Forward sequence (5'-3') } & \multicolumn{1}{c}{ Reverse sequence (5'-3') } & Product size (bp) \\
\hline CYTL1 & NM_018659 & AGATCACCCGCGACTTCA & GTAGTCACTGGGATTGGGTATT & 278 \\
$\beta$-actin & NM_001101.3 & TCATCACCATTGGCAATGAG & CACTGTGTTGGCGTACAGGT & 155 \\
\hline
\end{tabular}

Table II. Three siRNA sequences and their target sites in the CYTL1 gene.

\begin{tabular}{lccc}
\hline \multirow{2}{*}{ siRNA } & Sense strand & Antisense strand & $\begin{array}{c}\text { Target site of } \\
\text { CYTL1 mRNA (bp) }\end{array}$ \\
\hline siRNA1 & GAUUCCUUGAAGGACAAAGCACGGA & UCCGUGCUUUGUCCUUCAAGGAAUC & NM_018659: 293-317 \\
siRNA2 & GCUGUACACCAUCAUGAACUCGUUC & GAACGAGUUCAUGAUGGUGUACAGC & NM_018659: 319-343 \\
siRNA3 & GUUGGAUGACUGCAAUGCCUUGGAA & UUCCAAGGCAUUGCAGUCAUCCAAC & NM_018659:367-391 \\
\hline
\end{tabular}

were harvested and RNA was extracted for CYTL1 expression analysis as described below.

$N B$ specimens. Five NB tissue specimens with clinical stages III-IV were obtained from the Department of Surgery at the Beijing Children's Hospital in China. The patients were 1.4 to 7.6 years old (mean age, 5.3 years) and included 1 boy and 4 girls. NB tissues were snap-frozen at the time of surgery and stored at $-80^{\circ} \mathrm{C}$. RNA was isolated for real-time PCR analysis of CYTL1 expression as described below. The ethics committee of the Beijing Children's Hospital approved the protocol. Informed consent was obtained from the guardians of the patients.

RT-PCR analysis and quantitative real-time PCR (qRT-PCR). Total-RNA was isolated from the cultured cells and NB samples using the TRIzol reagent (Invitrogen, Carlsbad, CA, USA). One microgram of total-RNA was reverse transcribed in a $20 \mu \mathrm{l}$ reaction volume using oligo dT(15) primers and the M-MLV reverse transcriptase (Promega A3500, Madison, WI, USA). The levels of the CYTL1 and $\beta$-actin (internal control) transcripts were analyzed by PCR and real-time PCR. The primer sequences that were used are shown in Table I. The cycling conditions were designated as follows: an initial denaturation step at $95^{\circ} \mathrm{C}$ for $5 \mathrm{~min}$ followed by 35 cycles at $94^{\circ} \mathrm{C}$ for $1 \mathrm{~min}, 58^{\circ} \mathrm{C}$ for $30 \mathrm{sec}$ and $72^{\circ} \mathrm{C}$ for $20 \mathrm{sec}$. Amplified PCR products were analysed by electrophoresis on $1.2 \%$ agarose gels. For real-time PCR, cDNA was mixed with primers and SYBR-Green Supermix according to the manufacturer's protocols (Bio-Rad, Hercules, CA, USA). Real-time PCR was performed using a CHROMO4 continuous fluorescence detector (Bio-Rad) for relative quantitation of mRNA levels. For each sample, a relative quantity was calculated using the $2^{-\Delta} \mathrm{Ct}$ method (17).

Stealth siRNA-CYTL1 synthesis and transfection. Stealth siRNA-CYTL1 sequences were designed and synthesized by Life Technologies Corporation (Invitrogen). The sequences are shown in Table II. All of the sequences were analyzed using the Basic Local Alignment Search Tool (BLAST), available from the National Center for Biotechnology Information (http:// blast.ncbi.nlm.nih.gov/). The SH-SY5Y cells were divided into 6 groups: the mock control group (transfection reagent only), the negative control group (transfected with non-specific siRNA), the positive control group (transfected with Alexa Fluor Red Fluorescent Oligo) and 3 experimental groups (transfected with Stealth siRNA1-CYTL1, Stealth siRNA2-CYTL1, or Stealth siRNA3-CYTL1). Upon reaching 30 50\% confluency, the cells were transfected with siRNA using Lipofectamine RNAiMAX reagent (Invitrogen) according to the manufacturer's protocols. After transfection for $24 \mathrm{~h}$, the transfection efficiency of the positive control group was observed using a fluorescence microscope and analyzed by flow cytometry. Total-RNA was prepared from the samples collected at 24,48 and $72 \mathrm{~h}$ posttransfection and was used for RT-PCR and qRT-PCR. Total protein was extracted at $72 \mathrm{~h}$ post-transfection and used for western blot analysis.

Western blot analysis. After transfection for $72 \mathrm{~h}$, the cells were washed twice with PBS and then harvested with lysis buffer [20 mmol/1 Tris-HCl, pH 7.6; $100 \mathrm{mmol} / \mathrm{l} \mathrm{NaCl} ; 20 \mathrm{mmol} / 1$ $\mathrm{KCl} ; 1.5 \mathrm{mmol} / 1 \mathrm{MgCl}_{2}$ and $0.5 \%$ Nonidet $\mathrm{P}-40$ containing cocktail protease inhibitors (Roche)]. Total protein concentrations were determined using the BCA assay. Cell extracts were separated by $12 \%$ SDS-PAGE and transferred onto PVDF membranes (Millipore, Billerica, MA, USA) using standard techniques. The immobilized proteins were blocked with $5 \%$ skim milk in Tris-buffered saline/0.1\% Tween-20 (TBST), and then incubated with a 1:1,000 dilution of the primary antibody (anti-CYTL1, Sigma, St. Louis, MO, USA) at $4^{\circ} \mathrm{C}$ overnight. An HRP-conjugated IgG (KPL, Gaithersburg, MD, USA) was used as a secondary antibody ( $1 \mathrm{~h}$ incubation). The immunoreactive proteins were detected using the SuperSignal West Dura chemiluminescent detection reagent (Pierce, Rockford, IL, USA). The membranes were incubated with an anti-GAPDH antibody (Santa Cruz Biotechnology, Santa Cruz, CA, USA) as an internal control. All of the experiments were repeated 3 times with similar results. Blots were scanned using a Gel Doc 2000 Imaging System and the relative intensities of the proteins bands were normalized against GAPDH using Quantity One software (version 4.62, Bio-Rad). 
Cell counting kit-8 (CCK-8) assay. Cell proliferation and viability were determined by the CCK-8 assay (Dojindo, Kumamoto, Japan). The SH-SY5Y cells were seeded at a density of $1 \times 10^{4}$ cells per well in a 96-well culture plate. After incubation for $24 \mathrm{~h}$, the cells were transfected with Stealth siRNA-CYTL1 at a final concentration of $50 \mathrm{nM}$. After transfection for 0,24 , 48, 72 and $96 \mathrm{~h}, \mathrm{CCK}-8$ solution $(10 \mu \mathrm{l})$ was added to each well and the cells were incubated at $37^{\circ} \mathrm{C}$ for $4 \mathrm{~h}$. The optical density (OD) at $450 \mathrm{~nm}$ and $620 \mathrm{~nm}$ (reference wavelength) was measured using a spectrophotometer. In each sample, the normalized OD was defined as the OD at $450 \mathrm{~nm}$ minus the $\mathrm{OD}$ at $620 \mathrm{~nm}$. The inhibition of cell growth was calculated by comparing the normalized OD of the Stealth siRNA-CYTL1transfected SH-SY5Y cells with that of the negative control cells. Three independent experiments in quadruplicate wells were performed to verify the reproducibility of results.

Cell migration and invasion assays. Cell migration and invasion capabilities were measured in vitro using uncoated or growth factor reduced Matrigel-coated transwell inserts $(8 \mu \mathrm{m}$ pore size, BD Falcon, Franklin Lakes, NJ, USA) in 24-well plates. After transfection for $18 \mathrm{~h}$ with the Stealth siRNAs at a final concentration of $50 \mathrm{nM}$, cells $\left(5 \times 10^{4}\right.$ cells per well for the migration assay, or $1 \times 10^{5}$ cells per well for the invasion assay) were suspended in $200 \mu \mathrm{l}$ of DMEM without FBS and seeded onto the upper chambers of the transwells. The lower chambers of the transwells were filled with $500 \mu \mathrm{l}$ of DMEM containing $5 \%$ FBS (Hyclone). The chambers were then incubated at $37^{\circ} \mathrm{C}$ with $5 \% \mathrm{CO}_{2}$ for $10 \mathrm{~h}$ (migration assay) or $24 \mathrm{~h}$ (invasion assay). After incubation, the cells on the upper surface were carefully removed using a cotton swab. Cells that migrated or invaded through the filter to the lower surface were fixed with $4 \%$ paraformaldehyde for $20 \mathrm{~min}$ and stained with $0.1 \%$ crystal violet for $15 \mathrm{~min}$. Cells that migrated or invaded were visualised and photographed using a phase-contrast microscope (x400 magnification). Five fields of view per filter were counted; the fields were randomly chosen from the top, bottom, left, right, and center positions of each filter. Three independent experiments were performed in triplicate wells.

Statistical analysis. Results are presented as the mean \pm the standard deviation (SD). All of the statistical analyses were performed using the SPSS 11.5 software package for Windows. A one-way analysis of variance (ANOVA) was used for all comparisons. A value of $\mathrm{P}<0.05$ was defined as statistically significant, and a value of $\mathrm{P}<0.01$ was defined as highly statistically significant.

\section{Results}

CYTL1 is only expressed in the SH-SY5Y cell line and is highly expressed in NB tissues. To investigate if CYTL1 is involved in tumor pathogenesis, we examined the expression patterns of CYTL1 mRNA in 10 human tumor cell lines. RT-PCR analyses revealed that CYTL1 was only expressed in the SH-SY5Y neuroblastoma cell line (Fig. 1A), suggesting a potential role of CYTL1 specifically in NB development. To test this possibility, human NB tissues were collected and qRT-PCR was performed. As shown in Fig. 1B, high levels of expression of CYTL1 mRNA were observed in all 5 of the NB tissues. These results indicated that CYTL1 may play a specific role in NB development.
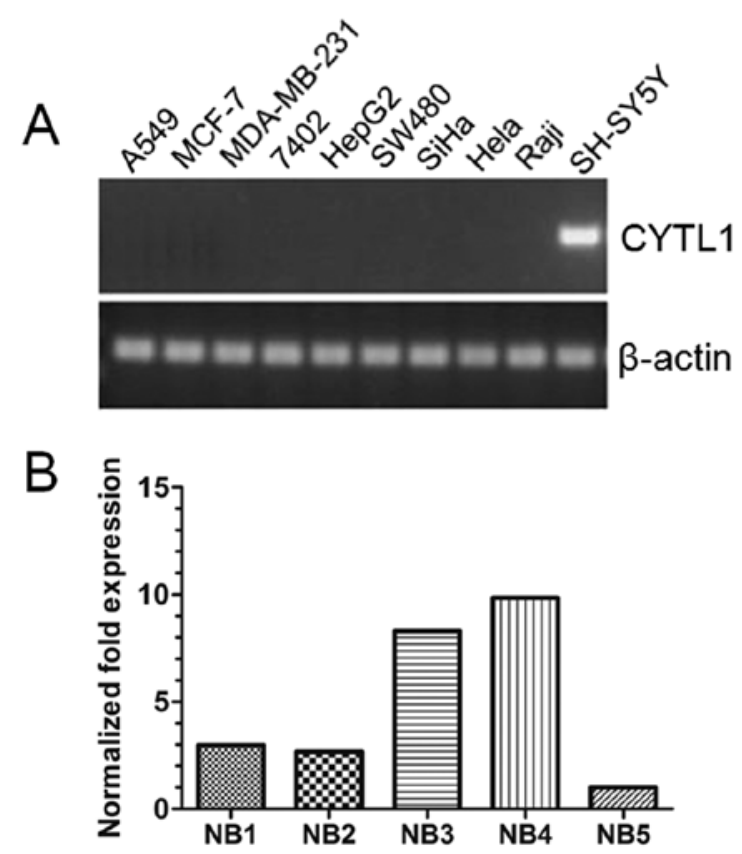

Figure 1. Expression of CYTL1 in human tumor cell lines and NB tissues CYTL1 mRNA expression was examined by RT-PCR or qRT-PCR in (A) 10 human tumor cell lines and in (B) 5 NB tissues. The relative levels of CYTL1 mRNA were normalized using $\beta$-actin as an internal control. The data in (A) and (B) are representative of three independent experiments.

Stealth siRNA-CYTL1 inhibits the mRNA and protein expression of CYTL1. To determine if and how CYTL1 may affect SH-SY5Y cells, we used Stealth siRNAs to knockdown CYTL1 expression in SH-SY5Y cells. Stealth siRNA-CYTL1 was transfected into SH-SY5Y cells. After transfection for $24 \mathrm{~h}$, the levels of Alexa Fluor Red Fluorescent Control were examined by fluorescence microscopy and flow cytometry. Transfection efficiency was greater than $85 \%$. Transient transfection of Stealth siRNA-CYTL1 resulted in knockdown rates of $44.2 \%$ (siRNA1), 66.1\% (siRNA2) and 93.7\% (siRNA3) within $24 \mathrm{~h}$. The knockdown effect of siRNA3-CYTL1 at the mRNA level was sustained for up to $72 \mathrm{~h}$ after transfection (Fig. 2). Western blot analysis showed that the levels of CYTL1 protein expression in the cells that were transfected with siRNA-CYTL1 were $15 \%$ (siRNA1), 35\% (siRNA2) and 80\% (siRNA3) lower than that of the negative control (NC) cells at $72 \mathrm{~h}$ after transfection (Fig. 3). The expression trends of CYTL1 protein and CYTL1 mRNA were roughly equivalent. These data demonstrated that Stealth siRNA3-CYTL1 efficiently knocked down CYTL1 expression. We therefore selected it for use in the subsequent experiments.

Knockdown of CYTL1 inhibits the growth of SH-SY5Y cells. We first examined whether CYTL1 regulates cell proliferation. The proliferation of SH-SY5Y cells was examined using the CCK-8 assay at 24, 48, 72 and $96 \mathrm{~h}$ after transfection. At $24 \mathrm{~h}$ after transfection, the proliferation of cells in the siRNA3-CYTL1 group was not significantly decreased compared with that of the NC group (7.91\% inhibition) (Fig. 4). However, the proliferation of SH-SY5Y cells in the siRNA3-CYTL1 group was significantly decreased compared with that of $\mathrm{NC}$ group at 48, 72 and $96 \mathrm{~h}$ after transfection $(24.16 \%, 28.36 \%$ and $39.33 \%$ inhibition, respectively, $\mathrm{P}<0.01$ ) (Fig. 4). There was no difference in prolif- 
A
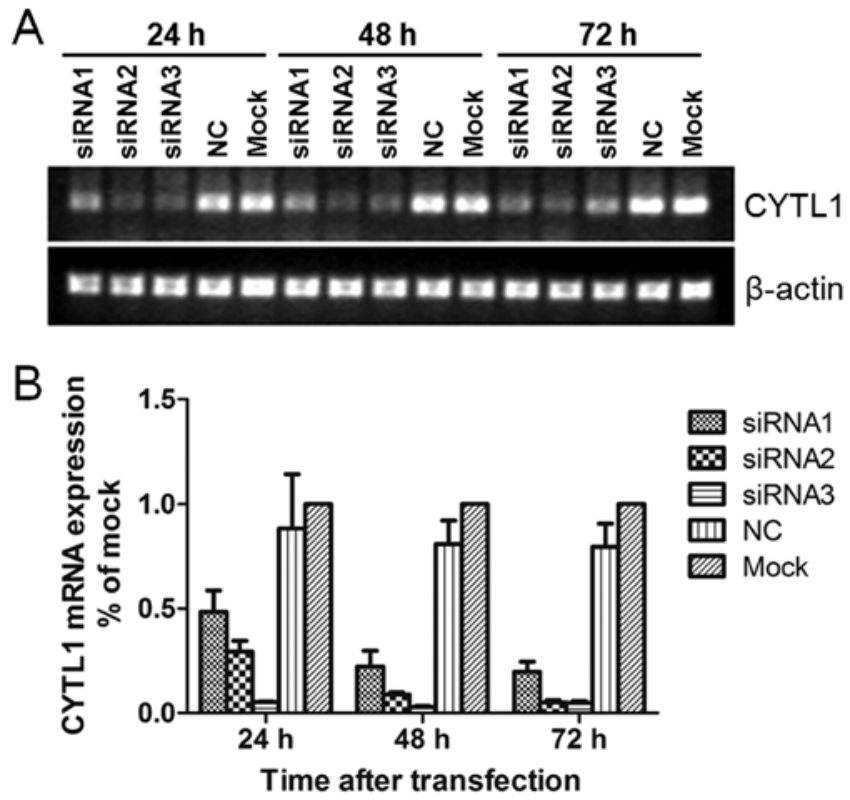

Figure 2. The expression of CYTL1 mRNA in SH-SY5Y cells was reduced by siRNA knockdown. CYTL1 mRNA expression was determined by (A) RT-PCR and (B) qRT-PCR at 24,48 and $72 \mathrm{~h}$ after transfection with Stealth siRNAs. The relative levels of CYTL1 mRNA were normalized using $\beta$-actin as an internal control. The data in (A) and (B) are representative of three independent experiments. NC, negative control; mock, no siRNA control.

A Si 1 Si 2 Si 3 NC Mock

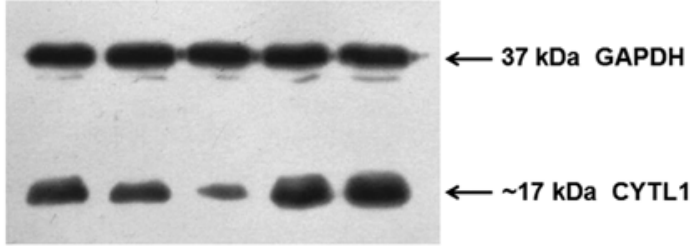

B

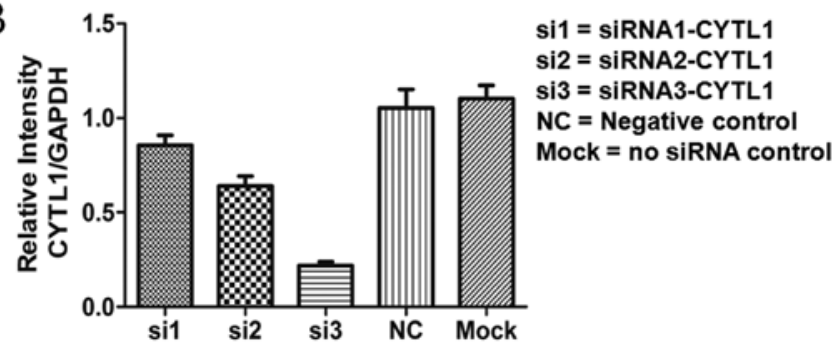

Figure 3. CYTL1 protein expression was decreased by siRNA knockdown in SH-SY5Y cells. CYTL1 protein expression was evaluated by (A) western blotting with lysates from cells that were treated with a $72 \mathrm{~h}$ transfection with Stealth siRNA-CYTL1, negative control, or mock control. (B) The relative intensities of the bands on the western blot were analyzed using Quantity One Software. Columns indicate the ratio of CYTL1 intensity to GAPDH intensity that was measured in three independent experiments.

eration between the $\mathrm{NC}$ group and the mock control group ( $\mathrm{P}>0.05)$. These results demonstrated that CYTL1 positively regulates the proliferation of SH-SY5Y cells.

Downregulation of CYTL1 results in decreased migration and invasion activities of SH-SY5Y cells. We next investigated whether CYTL1 plays a role in regulating the migration and

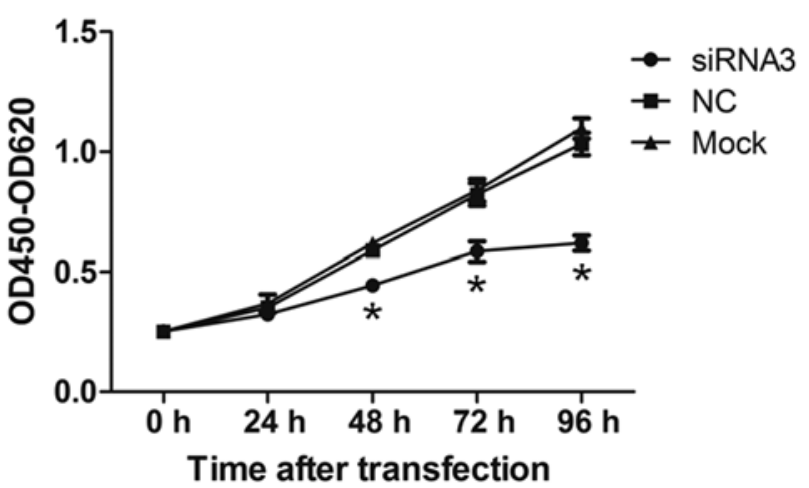

Figure 4. The proliferation of SH-SY5Y cells was measured by the CCK-8 assay. The value of OD450-OD620 for the siRNA3-CYTL1 group, NC group, and mock control group are shown for the indicated time points. Significant values are indicated by an asterisk $(\mathrm{P}<0.01$, comparison of the values of the siRNA3-CYTL1 group with those of the NC and mock control groups). siRNA3, siRNA3-CYTL1; NC, negative control; mock, no siRNA control.

invasion of SH-SY5Y cells. Migration and invasion assays were performed in transwell chambers that were either uncoated or coated with Matrigel. In the Stealth siRNA3-CYTL1 transfection group, the number of cells that crossed the membrane was significantly decreased compared with those in the $\mathrm{NC}$ group and the mock control group $(\mathrm{P}<0.01)$. The migration and invasion of the cells in the siRNA3-CYTL1 transfection group were decreased by $69.0 \%$ (migration assay) and $63.3 \%$ (invasion assay) compared with that of the $\mathrm{NC}$ group. There was no difference in migration or invasion between the $\mathrm{NC}$ group and the mock control group $(\mathrm{P}>0.05)$, indicating that neither the non-specific siRNA knockdown nor the transfection procedures affected cell migration and invasion (Fig. 5). Together, these results indicated that CYTL1 also plays an important role in regulating the migration and invasion activities of SH-SY5Y cells.

\section{Discussion}

Tumor metastasis is a complex multistep process including cell adhesion, cell migration, angiogenesis, immune evasion, and the homing of cells to target organs. Cell migration and invasion are essential features of the metastatic process (18-21). Recent research efforts have focused on the function of cytokines, chemokines, and their receptors in the growth and metastasis of NB (4-8,22). For example, CXCL12/CXCR4 and CCL2/CCR2 have recently become the subject of intense investigation $(5,23)$. A secondary structure prediction study previously showed that the structure of CYTL1 exhibits a unique 4-helical cytokine-fold (9). However, a more recent study using different computational structure-based techniques has proposed that CYTL1 is a putative chemokine with an IL-8-like structure similar to CCL2, which could signal through the CCR2 chemokine receptor (16). Therefore, we hypothesized that CYTL1 may contribute to the pathogenesis of NB by functioning as a CCL2-like chemokine.

In the present study, we assessed CYTL1 expression and function in SH-SY5Y cells. Our results demonstrated that CYTL1 was only expressed in the SH-SY5Y cells among the 10 human tumor cell lines that we examined. CYTL1 mRNA was also highly expressed in all 5 of the advanced stage NB tissues that we examined. Because nearly $70 \%$ of NB patients 


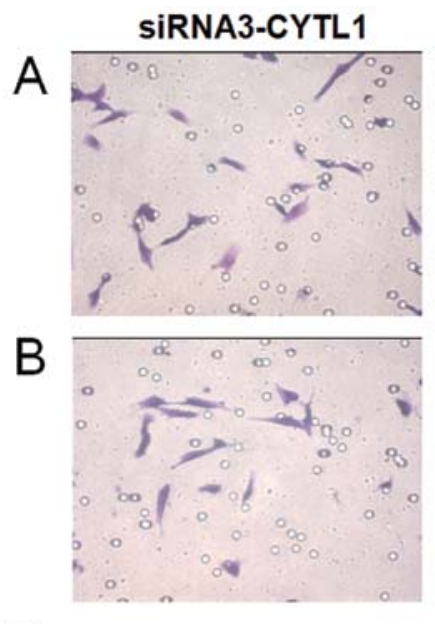

C

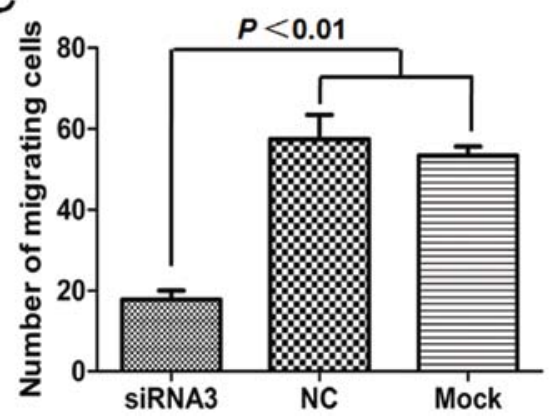

Negative control
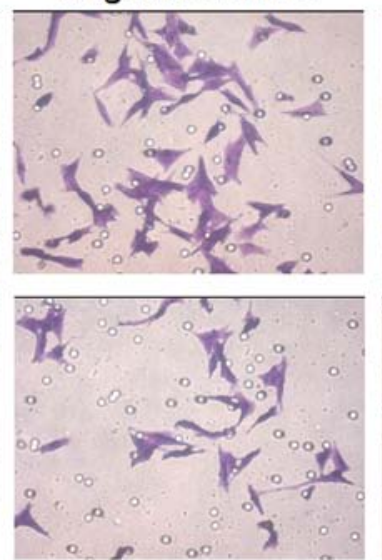

D

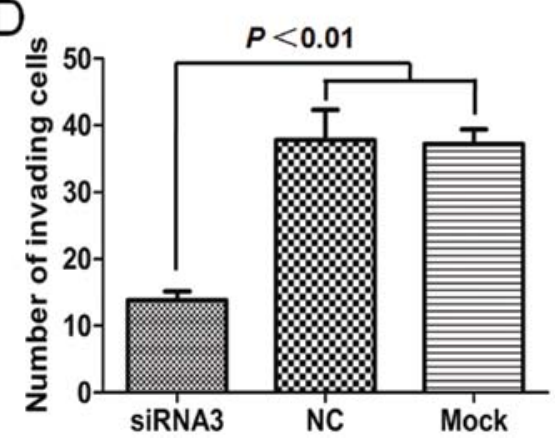

Figure 5. The migration and invasion activities of SH-SY5Y cells were measured using transwell assays. SH-SY5Y cells were transfected with Stealth siRNA3CYTL1 or negative control $(50 \mathrm{nM}, 18 \mathrm{~h})$ or mock control. The cells were next used in the migration assay (10 h) or the invasion assay ( $24 \mathrm{~h})$. The cells that migrated or invaded were stained with $0.1 \%$ crystal violet solution and visualized by microscopy. Representative images (magnification, $\mathrm{x} 400$ ) following (A) the migration and (B) invasion assays. The distribution of the number of (C) migrating or (D) invading cells in the siRNA3-CYTL1, NC and mock control groups. Columns represent the mean $\pm \mathrm{SD}$ of three independent experiments performed in triplicate. P $<0.01$, siRNA3-CYTL1 values compared with NC and mock control values.

already have metastatic disease at the time of primary diagnosis (24) it is difficult to collect early stage NB specimens. In the normal brain tissue of mice, however, the level of CYTL1 expression is very low (11). The SH-SY5Y cell line was derived from a site of NB metastasis in the bone marrow of a 4-year-old female suffering from NB. SH-SY5Y cells therefore represent a suitable model for studying the function of CYTL1 in NB. We evaluated the effects of altered CYTL1 expression on the proliferation, migration and invasion of SH-SY5Y cells with RNA interference (RNAi) technology.

RNAi refers to the inhibition of gene expression by small double-stranded RNA (dsRNA) molecules that target specific mRNAs for degradation. The ability of siRNAs to inhibit gene expression provides a novel tool for the genome-wide analysis of gene function and may represent a potentially useful therapeutic strategy. To date, many studies have demonstrated that RNAi-mediated gene silencing has promising therapeutic potential for the treatment of cancer (25-27). Studies have shown that siRNAs that target sequences more proximal to the $3^{\prime}$ end of the target gene may exhibit more efficient gene silencing effects; studies have also shown that the inhibitory efficiency of siRNAs depends on the format of the siRNA-longer, modified Stealth siRNAs generally are more efficient than standard siRNAs targeting the same sequence $(25,28,29)$. To achieve more effective knockdown and lower cytotoxicity, we used the Stealth siRNA and Lipofectamine RNAiMAX systems $(30,31)$ to deliver the siRNA duplexes into the SH-SY5Y cells. Our results revealed that Stealth siRNA3, which targeted the $3^{\prime}$ end of the CYTL1 mRNA (target site: NM_018659: 367-391 bp), had a higher knockdown efficiency than siRNA1 (target site: 293-317 bp) and siRNA2 (target site: 319-343 bp). Both the mRNA and protein levels of CYTL1 were markedly reduced in the SH-SY5Y cells that were transfected with Stealth siRNA3. Thus, Stealth siRNA3 was chosen for further study. Furthermore, the sequence targeted by siRNA3-CYTL1 may be a promising sequence to target for the knockdown of CYTL1 expression in NB therapy.

CYTL1 was first described in bone marrow-derived CD34 ${ }^{+}$ cells (9). Although knowledge of CYTL1 remains limited, previous studies have identified its involvement in the regulation of cartilage development and in benign prostatic hyperplasia. The expression and function of the CYTL1 gene in tumors remains unclear. A recent study of human lung squamous cell carcinoma (SCC) demonstrated that the expression of CYTL1 is regulated by DNA promoter methylation and suggested that CYTL1 may serve as a target molecule for the further study of SCC (15). In this study, we report for the first time the finding that CYTL1 is expressed in SH-SY5Y cells and in NB tissues. Under basal culture conditions, CYTL1 was highly expressed in SH-SY5Y cells. We therefore used a gene silencing strategy to effectively knock down its expression in SH-SY5Y cells. Using this strategy, we observed significant decreases in cellular proliferation, migration, and invasion, suggesting that CYTL1 
promotes the growth of SH-SY5Y cells and might contribute to NB progression.

In summary, our results provide the first evidence of CYTL1 expression in NB cell lines and tissues. Knockdown CYTL1 expression by Stealth siRNA demonstrated that CYTL1 positively regulates $\mathrm{NB}$ cell proliferation, migration and invasion in vitro. Collectively, we revealed a possible link between CYTL1 and NB development, discovered that CYTL1 is a new molecule that is involved in NB growth and metastasis, and suggested that CYTL1 may serve as a potential therapeutic target and diagnostic biomarker for NB.

\section{References}

1. McHugh K: Renal and adrenal tumours in children. Cancer Imaging 7: 41-51, 2007.

2. Zhu H, Zheng J, Xiao X, et al: Environmental endocrine disruptors promote invasion and metastasis of SK-N-SH human neuroblastoma cells. Oncol Rep 23: 129-139, 2010.

3. Rha SE, Byun JY, Jung SE, Chun HJ, Lee HG and Lee JM: Neurogenic tumors in the abdomen: tumor types and imaging characteristics. Radiographics 23: 29-43, 2003.

4. Pistoia V, Bianchi G, Borgonovo $G$ and Raffaghello L: Cytokines in neuroblastoma: from pathogenesis to treatment. Immunotherapy 3: 895-907, 2011.

5. Metelitsa LS, Wu HW, Wang H, et al: Natural killer T cells infiltrate neuroblastomas expressing the chemokine CCL2. J Exp Med 199: 1213-1221, 2004.

6. Zhang L, Yeger H, Das B, Irwin MS and Baruchel S: Tissue microenvironment modulates CXCR4 expression and tumor metastasis in neuroblastoma. Neoplasia 9: 36-46, 2007.

7. Zeng Y, Huebener N, Fest S, et al: Fractalkine (CX3CL1)- and interleukin-2-enriched neuroblastoma microenvironment induces eradication of metastases mediated by $\mathrm{T}$ cells and natural killer cells. Cancer Res 67: 2331-2338, 2007.

8. Raffaghello L, Cocco C, Corrias MV, Airoldi I and Pistoia V: Chemokines in neuroectodermal tumour progression and metastasis. Semin Cancer Biol 19: 97-102, 2009.

9. Liu X, Rapp N, Deans R and Cheng L: Molecular cloning and chromosomal mapping of a candidate cytokine gene selectively expressed in human CD34+ cells. Genomics 65: 283-292, 2000

10. Hermansson M, Sawaji Y, Bolton M, et al: Proteomic analysis of articular cartilage shows increased type II collagen synthesis in osteoarthritis and expression of inhibin betaA (activin A), a regulatory molecule for chondrocytes. J Biol Chem 279: 43514-43521, 2004.

11. Kim JS, Ryoo ZY and Chun JS: Cytokine-like 1 (Cytl1) regulates the chondrogenesis of mesenchymal cells. J Biol Chem 282: 29359-29367, 2007.

12. Jeon J, Oh H, Lee G, et al: Cytokine-like 1 knock-out mice (Cytl1-/-) show normal cartilage and bone development but exhibit augmented osteoarthritic cartilage destruction. J Biol Chem 286: 27206-27213, 2011.
13. Chao C, Joyce-Shaikh B, Grein J, et al: C17 prevents inflammatory arthritis and associated joint destruction in mice. PLoS One 6: e22256, 2011.

14. Begley LA, Kasina S, MacDonald J and Macoska JA: The inflammatory microenvironment of the aging prostate facilitates cellular proliferation and hypertrophy. Cytokine 43: 194-199, 2008.

15. Kwon YJ, Lee SJ, Koh JS, et al: Genome-wide analysis of DNA methylation and the gene expression change in lung cancer. J Thorac Oncol 7: 20-33, 2012.

16. Tomczak A and Pisabarro MT: Identification of CCR2-binding features in Cytl1 by a CCL2-like chemokine model. Proteins 79: 1277-1292, 2011.

17. Livak KJ and Schmittgen TD: Analysis of relative gene expression data using real-time quantitative PCR and the $2-\Delta \Delta C T$ method. Methods 25: 402-408, 2001.

18. Thiery JP and Sleeman JP: Complex networks orchestrate epithelial-mesenchymal transitions. Nat Rev Mol Cell Biol 7: 131-142, 2006.

19. Chambers AF, Groom AC and MacDonald IC: Dissemination and growth of cancer cells in metastatic sites. Nat Rev Cancer 2: 563-572, 2002.

20. Pienta KJ and Loberg R: The 'emigration, migration, and immigration' of prostate cancer. Clin Prostate Cancer 4: 24-30, 2005.

21. Vicari AP and Caux C: Chemokines in cancer. Cytokine Growth Factor Rev 13: 143-154, 2002.

22. Gross N and Meier R: Chemokines in neuroectodermal cancers: the crucial growth signal from the soil. Semin Cancer Biol 19: 103-110, 2009

23. Geminder H, Sagi-Assif O, Goldberg L, et al: A possible role for CXCR4 and its ligand, the CXC chemokine stromal cellderived factor-1, in the development of bone marrow metastases in neuroblastoma. J Immunol 167: 4747-4757, 2001.

24. Ara T and DeClerck YA: Mechanisms of invasion and metastasis in human neuroblastoma. Cancer Metastasis Rev 25: 645-657, 2006.

25. Elbashir SM, Harborth J, Weber K and Tuschl T: Analysis of gene function in somatic mammalian cells using small interfering RNAs. Methods 26: 199-213, 2002.

26. Zamore PD: RNA interference: listening to the sound of silence. Nat Struct Biol 8: 746-750, 2001.

27. Burnett JC, Rossi JJ and Tiemann K: Current progress of siRNA/shRNA therapeutics in clinical trials. Biotechnol J 6: 1130-1146, 2011.

28. Patel R, T'Wallant N C, Herbert MH, White D, Murison JG and Reid G: The potency of siRNA-mediated growth inhibition following silencing of essential genes is dependent on siRNA design and varies with target sequence. Oligonucleotides 19: 317-328, 2009.

29. Suggate EL, Ahmed Z, Read ML, et al: Optimisation of siRNA-mediated RhoA silencing in neuronal cultures. Mol Cell Neurosci 40: 451-462, 2009.

30. Zhao M, Yang H, Jiang X, et al: Lipofectamine RNAiMAX: an efficient siRNA transfection reagent in human embryonic stem cells. Mol Biotechnol 40: 19-26, 2008.

31. Nabzdyk CS, Chun M, Pradhan L and Logerfo FW: High throughput RNAi assay optimization using adherent cell cytometry. J Transl Med 9: 48, 2011. 